\title{
DIFFUSION TEMPERATURE DEPENDENCE IN TILTED PERIODIC POTENTIALS RANGING FROM UNDERDAMPED SYSTEMS TO OVERDAMPED SYSTEMS
}

\author{
(DI.G. Marchenko ${ }^{1,2 *}$, (DI.I. Marchenko3, (DV.I. Tkachenko ${ }^{1,2}$ \\ ${ }^{\prime}$ National Science Center ,Kharkov Institute of Physics and Technology” \\ 1 Akademicheskaia Street, Kharkov 61108 Ukraine \\ ${ }^{2}$ V.N. Karazin Kharkov National University \\ 4 Svobody Square, Kharkov 61022 Ukraine \\ ${ }^{3} N T U$ "Kharkov Polytechnic Institute" \\ 2 Kirpicheva St., Kharkov, 61002, Ukraine \\ *E-mail:march@kipt.kharkov.ua \\ Received 25 October 2018, accepted 16 November 2018
}

Recently, both the experimental data and the data of theoretical research appeared stating that the diffusion coefficient shows nonmonotonic behavior with temperature. The motion of Brownian particles in the space periodic structures is an example of the systems with abnormal temperature dependence of the diffusion. The aim of the work was to study the change in the temperature dependence of the diffusion coefficient with a change in friction, both in underdamp and overdamped systems. This scientific paper studies the diffusion of particles in tilted spatial-periodic potentials in a wide temperature range. It is shown that in both underdempted and overdamped systems, the diffusion coefficient reaches a maximum value for a certain value of an external force, the value of which depends on the value of the friction coefficient. However, in systems with low and high friction, the temperature dependence of the diffusion coefficient differs. It was established that the systems with a low friction level $\gamma$ ' show temperature abnormal diffusion (TAD) at which the diffusion coefficient $\mathrm{D}$ is increased with a decrease in temperature. At the same time, the diffusion is enhanced at high $\gamma$ values with the rise in temperature. This scientific paper studies the transition procedure from the exponential dependence of TAD to the ordinary power temperature dependence with an increase in $\gamma$ '. It was shown that the energy hump that separates "running" solutions and "localized" solutions is decreased with an increase in the friction coefficient and it vanishes at $\gamma^{\prime} \rightarrow 2$. Simultaneously with a decrease in $\varepsilon$, the temperature interval of the TAD also narrows. It was established that the temperature-limited TAD domain appears in the region of intermediate values of the friction coefficient. In a specified force range the diffusion coefficient is first increased with a decrease in temperature and then it begins to decrease again. The diagrams of existence of such domains have been constructed. The results obtained opens up prospects for the creation of new technologies for managing diffusion processes. This is of great importance for the production of nanomaterials with a given structure, the creation of surface nanostructures, etc.

KEY WORDS: diffusion, computer simulation, periodic structures, Langevin equation, time-periodic fields

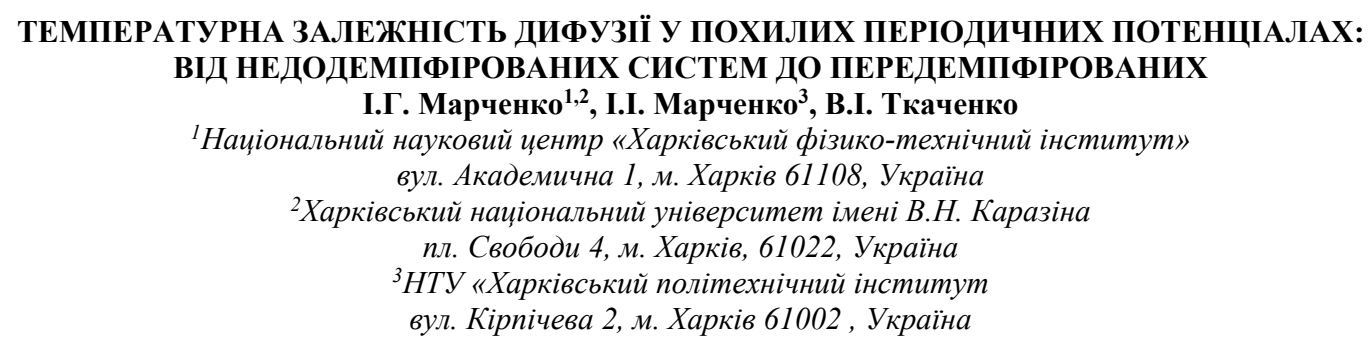

Останнім часом з'явилися як експериментальні дані, так і теоретичні дослідження, в яких коефіцієнт дифузії поводиться немонотонно 3 температурою. Одним із прикладів систем 3 аномальною температурної залежністю дифузії $\epsilon$ рух броунівських часток в просторово-періодичних структурах. Метою роботи було дослідження зміни температурної залежності коефіцієнта дифузії зі зміною тертя, як в недодемпфірованих, так і передемпфірованих системах. У роботі досліджена дифузія частинок у похилих просторово-періодичних потенціалах у широкому діапазоні температур. Показано, що як в недодемпфірованих, так і в передемпфірованих системах, коефіцієнт дифузії досягає максимального значення при певному значенні зовнішньої сили, величина якої залежить від величини коефіцієнта тертя. Однак в системах 3 малим $\mathrm{i}$ великим тертям температурна залежність коефіцієнта дифузії відрізняється. Показано, що у системах з малим рівнем тертя $\gamma^{\prime}$ спостерігається температурно-аномальна дифузія (ТАД), при якій коефіцієнт диффузії зростає із зниженням температури. У той же час при великих значеннях $\gamma^{\prime}$ дифузія посилюється із зростанням температури. У роботі досліджено яким чином відбувається переход від експоненціальної залежності ТАД до звичайної степенної температурної залежності із зростанням $\gamma$ '. Показано, що із збільшенням коефіцієнту тертя енергетичний барєр $\varepsilon$, що розділяє “локалізовані” та “бігучі” рішення зменшується, зникаючи при $\gamma^{\prime} \rightarrow 2$. Одночасно із зменьшенням $\varepsilon$ також відбувається звуження температурного інтервалу температурно-аномальної дифузії. Встановлено, що при проміжних значеннях коефіцієнту тертя виникає область температурно обмеженої ТАД. У деякому інтервалі сил коєфіцієнт дифузії спочатку зростає із зниженням температури, а потім знову починає падати. Побудовані диаграми існування таких областей. Отримані результати відкривають перспективи створення нових технологій керування процесами дифузії. Це має велике значення для отримання наноматеріалів із заданою структурою, створення поверхневих наноструктур та ін.

КЛЮЧОВІ СЛОВА: дифузія, комп'ютерне моделювання, броуновський рух, періодичні структури, рівняння Ланжевена (C) Marchenko I.G., Marchenko I.I., Tkachenko V.I., 2018 


\section{ТЕМПЕРАТУРНАЯ ЗАВИСИМОСТЬ ДИФФУЗИИ В ПЕРИОДИЧЕСКИХ НАКЛОННЫХ ПОТЕНЦИАЛАХ: ОТ НЕДОДЕМПФИРОВАНЫХ СИСТЕМ К ПЕРЕДЕМПФИРОВАНЫМ}

\section{И.Г. Марченко ${ }^{1,2}$, И.И. Марченко ${ }^{3}$, В.И. Ткаченко ${ }^{1,2}$}

${ }^{1}$ Национальний научный центр «Харьковский физико-технический институт» ул. Академическая 1, г. Харьков 61108, Украина

${ }^{2}$ Харьковский национальный университет имени В.Н. Каразина пл. Свободьл 4, г. Харьков, 61022, Украина

${ }^{3}$ НТУ «Харьковский политехнический институт

ул. Кирпичева 2, г. Харьков 61002, Украина

В последнее время появились как экспериментальные данные, так и теоретические исследования, в которых коэффициент диффузии ведет себя немонотонно с температурой. Одним из примеров систем с аномальной температурной зависимостью диффузии является движение броуновских частиц в пространственно-периодических структурах. Целью работы было исследование изменения температурной зависимости коэффициента диффузии с изменением трения, как в недодемпфированых, так и передемпфированых системах. В работе изучена диффузия частиц в наклонных пространственно-периодических потенциалах в широком диапазоне температур. Показано, что как в недодемпфированых, так и в передемпфированых системах, коэффициент диффузии достигает максимального значения при определенном значении внешней силы, величина которой зависит от величины коэффициента трения. Однако в системах с малым и большим трением температурная зависимость коэффициента диффузии различается. Показано, что в системах с малым уровнем трения $\gamma$ ' наблюдается температурно-аномальная диффузия (ТАД) при которой коэффициент диффузии D возрастает с понижением температуры. В то же время при больших значениях $\gamma$ ' диффузия усиливается с ростом температуры. В работе исследовано, каким образом с ростом $\gamma$ ' осуществляется переход от экспоненциальной зависимости ТАД к обычной степенной температурной зависимости. Показано, что с увеличением коэффициента трения энергетический барьер $\varepsilon$, разделяющий “бегущие” и “локализованные” решения, уменьшается, исчезая при $\gamma^{\prime} \rightarrow 2$. Одновременно с уменьшением $\varepsilon$ также происходит сужение температурного интервала температурно-аномальной диффузии. Установлено, что при промежуточных значениях коэффициента трения возникает область температурно-ограниченной ТАД. В некотором интервале сил коэффициент диффузии сначала возрастает с уменьшением температуры, а затем снова начинает падать. Построены диаграммы существования таких областей. Полученные результаты открывает перспективы создания новых технологий управления процессами диффузии. Это имеет большое значение для получения наноматериалов с заданной структурой, создания поверхностных наноструктур и пр.

КЛЮЧЕВЫЕ СЛОВА: диффузия, компьютерное моделирование, броуновское движение, периодические структуры, уравнения Ланжевена.

Наше интуитивное представление о диффузии говорит нам, что с увеличением температуры диффузия растет. Вместе с тем это утверждение несправедливо для систем, далеких от равновесия. В последнее время появились как экспериментальные данные, так и теоретические исследования, в которых коэффициент диффузии ведет себя немонотонно с температурой [1-17]. Одним из примеров систем с аномальной температурной зависимостью диффузии является движение броуновских частиц в пространственнопериодических структурах. Решение задачи о движении таких частиц связано с широким кругом вопросов физики, химии и биологии. Это не только классическая диффузия в кристаллах, но и электропроводность в контактах Джозефсона, распространение волн зарядовой плотности, проницаемость мембран, процессы в суперионных проводниках и многое другое [18].

При теоретическом рассмотрении задачи диффузии броуновских частиц в вязкой среде с безразмерным коэффициентом трения $\gamma^{\prime}$ обычно рассматривают два предельных случая: случай недодемпфированого движения $\left(\gamma^{\prime}<<1\right)$ и передемпфированого движения $\left(\gamma^{\prime}>>1\right)$. Транспорт и диффузия частиц в периодическом потенциале впервые систематически был исследован Х. Рискеном [19-24], как для случая недодемпфированного, так и для передемпфированного движения. Было показано, что для недодемпфированного случая важным в поведении ансамбля частиц является возникновение “локализованных" и “бегущих" решений. При внешней силе F превышающей критическое значение $\mathrm{F}_{\mathrm{cr}}$ возникает бифуркация и вместо одного решения, возникает два: так называемые “локализованное” и “бегущее” решения. Обобщение результатов работ [19-24] можно найти в монографии [18]. Х. Рискеном были получены функции распределения частиц и выражения для мобильности частиц. В то же время поведение коэффициента диффузии не было исследовано, что в определенной мере связано с плохой применимостью используемых автором аналитических методов решения уравнения Фокера-Планка к системам с малой диссипацией из-за слабой сходимости решения. Альтернативным способом изучения процессов диффузии частиц является прямое численное моделирования движения частиц с помощью стохастических уравнений Ланжевена. Диффузия в периодических наклонных потенциалах систематически исследовались Ф. Марчезони и др. в одной из первых работ по численному решению этих уравнений [25-26]. Ими был установлен существенный рост пространственной диффузии частиц в системах с малой диссипацией вблизи критической силы, вызванный переходом частиц из “локализованного" в “бегущее” решение. Дальнейшее изучение диффузии под воздействием постоянной силы было связано с работами группы К. Линденберг [27-30]. Было установлено существование особенности в температурной зависимости диффузии для недодемпфированых систем. В работе [30] впервые было показано, что в наклонных периодических потенциалах коэффициент диффузии D ведет себя аномальным образом. При некотором значении силы он рос с понижением температуры. В работах [31-32] 
было показано, что в недодемпфированых системах диффузия возрастает с понижением температуры экспоненциальным образом: $D_{\max } \propto \exp (\varepsilon / k T)$ в определенном интервале приложенных внешних сил. Этот явление авторы назвали температурно-аномальной диффузией (ТАД) [33-34]. Феномен ТАД связан со слабым нарушением эргодичности в таких системах, поскольку время корреляции экспоненциально стремится к бесконечности при $T \rightarrow 0$. С падением температуры переход частиц из “бегущих" в “локализованные” решения и обратно происходит с меньшей частотой. Вследствие этого длина “скачков” броуновских частиц увеличивается. В работе [33] была построена феноменологическая модель, объясняющая такое поведение частиц.

С другой стороны, в случае передемпфированых систем, движение частиц в синусоидальном потенциале не демонстрирует аномальной температурной зависимости. Для передемпфированого случая Риман и соавторы [35] показали, что максимальное усиление диффузии происходит при некоторой критической силе. Им аналитически было показано, что с температурой максимальный коэффициент диффузии в синусоидальном потенциале растет, как $D_{\max } \propto T^{1 / 3}$. То есть при больших $\gamma$ температурная зависимость не демонстрирует аномальности. Однако неясно, каким образом функциональная зависимость $D_{\max }$ для синусоидального потенциала изменяется с аномальной $\left(D_{\max } \sim \exp (\varepsilon / k T)\right)$ на нормальную температурную зависимость $\left(D_{\max } \propto T^{1 / 3}\right)$.

Целью работы было исследование изменения температурной зависимости коэффициента диффузии с изменением трения, как в недодемпфированых, так и передемпфированых системах. Для этого в первой части работы мы изучим изменение $D(T)$ для недодемпфированных систем с $\gamma<0,03$. Во второй части мы проанализируем температурную зависимость диффузии для значений $\gamma>2$. В третьей части мы рассмотрим переходные значения $0.1<\gamma<2$, и исследуем как изменяется температурная зависимость диффузии с ростом $\gamma$. В заключении работы мы проанализируем как изменяется ширина интервала сил в котором наблюдается ТАД с ростом $\gamma$, а так же установим предельное значение $\gamma$, при котором наблюдается ТАД.

\section{МЕТОДИКА МОДЕЛИРОВАНИЯ}

Движение броуновских частиц в наклонном периодическом потенциале описывалось уравнением Ланжевена:

$$
m \ddot{x}=-\frac{\partial}{\partial x} U(x)-\gamma \dot{x}+F+\xi(t)
$$

где $t$ - время, $x$ - координата частицы в одномерной решетке, $m$ - ее масса, $\gamma$ - коэффициент трения, $F$ действующая на частицу постоянная сила, задающая наклон потенциала. Точка сверху означает дифференцирование по времени. Член $\xi(t)$ описывает термические флуктуации. Шум является белым гауссовым и соответственно для термических флуктуаций выполняется соотношение:

$$
\left.\xi(t) \xi\left(t^{\prime}\right)\right\rangle=2 \not k T \delta\left(t-t^{\prime}\right),
$$

где $k$ - постоянная Больцмана, $T$ - температура.

Потенциальная энергия частицы $U$ была равна:

$$
U(x)=-\frac{U_{0}}{2} \cos \left(\frac{2 \pi}{a} x\right),
$$

где $a$ - период одномерной решетки, а $U_{0}$ - высота потенциального барьера.

На движущуюся частицу, помимо постоянной внешней силы, действует пространственно-периодическая сила $F_{\text {lat }}$ :

$$
F_{l a t}=-\frac{\partial U}{\partial x}=F_{0} \sin \left(\frac{2 \pi}{a} x\right)
$$

Величина $F_{0}=\frac{\pi}{a} U_{0}$, называется критической силой [36-37]. Она соответствует минимальной силе, необходимой для преодоления в вязкой среде энергетического барьера, разделяющего два соседних положения частицы на решетке. Параметры используемого пространственно-периодического потенциала были теми же, что и в работах [20-22,38]: $U_{0}=0,08$ эВ $, a=2,0 \AA \AA$. Масса частиц соответствовала массе водорода и была равна 1 атомной единице массы. 
Стохастические уравнения (1)-(2) для каждой частицы решались численно с шагом по времени, составляющим менее 0.01 периода собственных колебаний $\tau_{0}=a\left(2 m / U_{0}\right)^{1 / 2}$. Статистическое усреднение проводилось по ансамблю с количеством частиц не менее $N=5 \cdot 10^{4}$. Для проверки полученных результатов отдельные вычисления проводились с $N=5 \cdot 10^{6}$. Начальные условия задавались следующим образом. Частица помещалась в начале координат и ей случайным образом сообщалась скорость, имеющая Максвелловское распределение по температуре. Для достижения равновесной функции распределения частиц, как по скоростям, так и по координатам, проводилась термализация системы в течение $10^{4}$ временных шагов. Как показали расчеты, после этого времени распределение ансамбля частиц, как по координатам, так и по скоростям не менялось. В процессе термализации частицы могли совершать скачок в соседние элементарные ячейки одномерной решетки. Для того чтобы диффузия частиц происходила из начала координат, такие частицы перемещалась в первую элементарную ячейку путем трансляции на целое количество постоянных решетки.

При анализе результатов моделирования удобно перейти к безразмерным величинам. Наиболее часто используются безразмерные величины, введенные Х. Рискеном [18], при которых уравнение (1) принимает максимально простой вид:

$$
\ddot{x}=-\sin x-\gamma^{\prime} \dot{x}+F+\xi(t)
$$

При этом выборе единиц высота энергетического барьера $U_{0}=2$, пространственный период потенциала $a=2 \pi$ и масса частиц $m=1$. Период собственных малых колебаний равен $\tau_{0}^{\prime}=2 \pi$. В этой системе единиц коэффициент трения $\gamma$ ' связан с $\gamma$ следующим образом:

$$
\gamma^{\prime}=\gamma \frac{\tau_{0}}{\tau_{o}^{\prime}}
$$

Для описания характера колебаний в различных системах обычно вводят коэффициент затухания [39]: $\zeta=\frac{\gamma}{2 m \omega_{0}}$, где $\omega_{0}$ - частота собственных колебаний. При $0<\zeta<1$ колебания называют недодемпфироваными, а при $\zeta>1$ - передемфпироваными. В случае $\zeta=1$ говорят о критическом демпфировании. Для уравнения (5) критическое демпфирование малых колебаний частиц около положения равновесия будет происходить при $\gamma^{\prime}=2$. Поэтому далее будем исследовать движение броуновских частиц в двух различных диапазонах: недодемпфированом ( $0<\gamma^{\prime}<2$ ) и передемпфированом $\left(\gamma^{\prime}>2\right)$.

Далее мы также будем использовать безразмерную величину температуры $T^{*}$ :

$$
T^{*}=\frac{T k}{U_{0}}=T^{\prime} / 2
$$

где $T^{\prime}$ - безразмерная величина температуры, используемая Х. Рискеном.

Коэффициент диффузии вычислялся по дисперсии $\sigma^{2}$ в распределении ансамбля движущихся частиц при стремлении времени к бесконечности:

$$
D=\lim _{t \rightarrow \infty} D_{e f}(t)=\lim _{t \rightarrow \infty} \frac{\left\langle\left(x-\langle x)^{2}\right\rangle\right.}{2 t}=\lim _{t \rightarrow \infty} \frac{\sigma^{2}}{2 t},
$$

где скобки $\langle.$.$\rangle обозначают усреднение по ансамблю. При каждом расчете коэффициента диффузии$ определялось время $t_{\text {lin }}$ достижения линейной зависимости дисперсии от времени. Коэффициент диффузии определялся по методу наименьших квадратов при времени $t>100 t_{\text {lin }}$ путем подгонки коэффициента пропорциональности дисперсии времени.

В работах [20-22] было показано, что в недодемпфированных системах с синусоидальным потенциалом существует интервал значений сил (зона ТАД), зависящий от коэффициента трения, при котором D бесконечно возрастает с понижением температуры. Вместе с тем для периодического воздействия [34] было установлено, что такой рост коэффициента диффузии наблюдается только в ограниченном интервале температур. И. Соколов и Б. Линдер путем численной обработки данных моделирования пришли к выводу, что и при постоянной силе возможно существование ТАД в ограниченном интервале температур [37]. Поэтому для универсальности мы в дальнейшем областью температурно-аномальной диффузии (ТАД) будем называть область параметров (Т,F, $\gamma$ ), 
в которой частная производная $\frac{\partial D(T, F, \gamma)}{\partial T}$ меньше нуля. Ширину температурного интервала ТАД для фиксированных значениях $\gamma$ и $F$ обозначим как $\Delta T(F, \gamma)=T_{m x}(F, \gamma)-T_{\min }(F, \gamma)$. Ширину силового интервала, в котором для какой-нибудь температуры $\frac{\partial D(T, F, \gamma)}{\partial T}<0$ при фиксированном $\gamma$ обозначим как $\Delta F(\gamma)$.

\section{НЕДОДЕМПФИРОВАННЫЕ СИСТЕМЫ $\left(\gamma^{\prime}<0.03\right)$}

Нами было проведено численное моделирование уравнения (1) для различных значений коэффициентов трения и температур. На рис. 1 представлены зависимости коэффициентов диффузии от силы для низких температур $\left(T^{*}<1\right)$. Величина $D_{0}=\pi^{2} / \tau_{0}$, где $\tau_{0}=a \sqrt{2 m / U_{0}}$ - период собственных малых колебаний около положения равновесия в потенциальном поле $U(X)$. Три группы графиков, обозначенные цифрами $1-3$, соответствуют различным коэффициентам трения. Из вида группы графиков следует, что положение и ширина силового интервала $\Delta F\left(\gamma^{\prime}\right)$ линейно уменьшаются с уменьшением $\gamma^{\prime}$. Детальный анализ всех данных показывает, что при этом максимальное значение D линейно растет с уменьшением $\gamma$ '. Чтобы понять физические причины такого поведения, проанализируем изменение функции распределения частиц по скоростям $n(V)$ с изменением $\mathrm{F}$ и $\gamma^{\prime}$. На рис. 2 в качестве примера приведены графики функций $n(V)$ для двух значений сил: $F / F_{0}=0,01$ и $F / F_{0}=0,001$ при различных $\gamma^{\prime}$, которые так же отличались на порядок $\left(\gamma^{\prime}=\right.$ $3 \cdot 10^{-3}$ и $\left.\gamma^{\prime}=3 \cdot 10^{-4}\right), V_{0}=U_{0} / m$. Как следует из рис. 2, при использованных значениях $\mathrm{F}$ и $\gamma^{\prime}$ функции $n\left(V ; F^{\prime}, \gamma^{\prime}\right)$ совпадают.

Из анализа графиков (рис. 2) и других подобных ему зависимостей $n\left(V ; F, \gamma^{\prime}\right)$ можно сделать вывод, что при малых $\gamma^{\prime}$ функция распределения по скоростям зависит только от отношения $\mathrm{F} / \gamma$. Впервые это было отмечено в монографии Х. Рискена (рис. 11.22-11.22а [18]). Из того факта, что $n(V, F, \Gamma)=n(V, F / \Gamma)$, можно получить скэйлинговые зависимости для коэффициентов диффузии [33]:

$$
D_{1}\left(\Gamma_{1}, F_{1}\right) / \Gamma_{1}=D_{2}\left(\Gamma_{2}, F_{1} \Gamma_{2} / \Gamma_{1}\right) / \Gamma_{2} .
$$

На рис. 3 приведены графики зависимости коэффициентов диффузии от действующей силы для различных коэффициентов трения. Моделирование проводилось при трех различных значениях температуры: $T^{*}=0,13 ; 0,19 ; 0,39$. На рис. 3 область ТАД, в которой коэффициент диффузии растет с понижением температуры, выделен штриховкой. Из представленных графиков следует, что скейлинговое соотношение (10) выполняется с хорошей точностью.

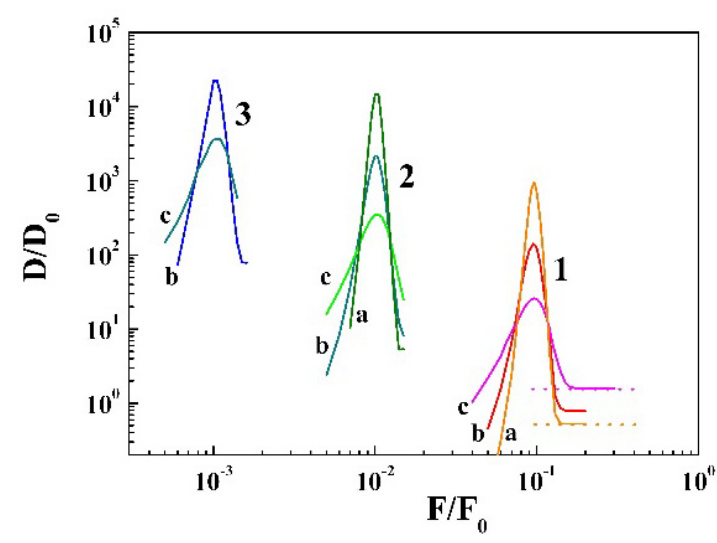

Рис.1. Зависимости коэффициентов диффузии от действующей силы для различных $\gamma$ '

$1-\gamma=3 \cdot 10^{-2}, 2-\gamma^{\prime}=3 \cdot 10^{-3} 3-\gamma^{\prime}=3 \cdot 10^{-4}$.

$D_{0}=\pi^{2} / \tau_{0}$. Температуры равны: а $-T^{\prime}=0,13$, b - $T^{\prime}=0,19, \mathrm{c}-T^{\prime}=0,39$.

Пунктирными линиями показаны коэффициенты диффузии в вязкой среде: $D=k T / \gamma$.

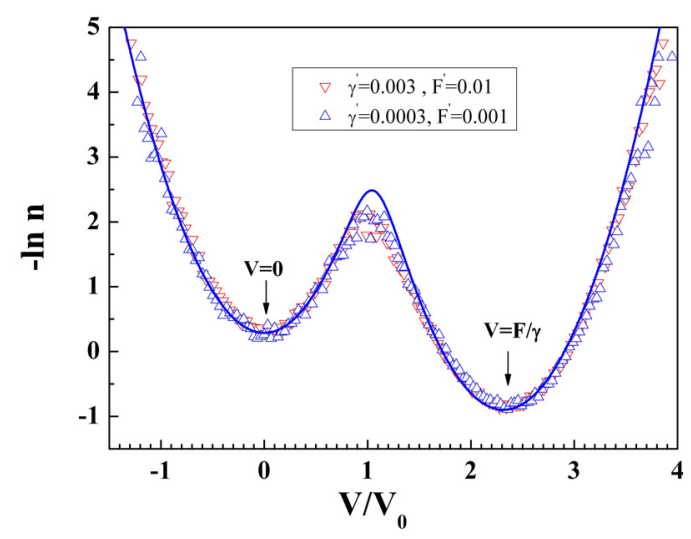

Рис. 2. Функции распределения частиц по скоростям $n(V)$ при значениях силы $F_{m x}(\gamma)$ когда достигается максимум $D(F)$.

$T^{\prime}=0,19, \nabla-\gamma^{\prime}=3 \cdot 10^{-3}, \Delta-\gamma^{\prime}=3 \cdot 10^{-4}$. Сплошной линией нанесены расчетные значения $n(V)$, исходя из модели двухъямного потенциала $W(V)$ [32]. Функция имеет минимумы при $V=0$ и $V=F / \gamma$

Вместо величины скорости $V_{0}=\left(U_{0} / m\right)^{1 / 2}$ на графиках рис. 3 используется значение $V_{F}$. Это связано с тем, что под действием силы изменяется величина энергетического барьера, который преодолевают частицы. 
Он зависит от наклона потенциального рельефа $F$. Однако, с изменением $\gamma$, интервал сил, ограничивающих область ТАД уменьшался $\propto \gamma$. Соответственно, при стремлении коэффициента трения к нулю величина энергетического барьера стремится к $U_{0}$. Поэтому различия в графиках должно нивелироваться с уменьшением $\gamma$. Графики на рис. 3, соответствующие температуре $T^{*}=0,19$, показывают, что это действительно так. Кривые, соответствующие $\gamma^{\prime}=0,003$ и $\gamma^{\prime}=0,0003$, практически совпадают.

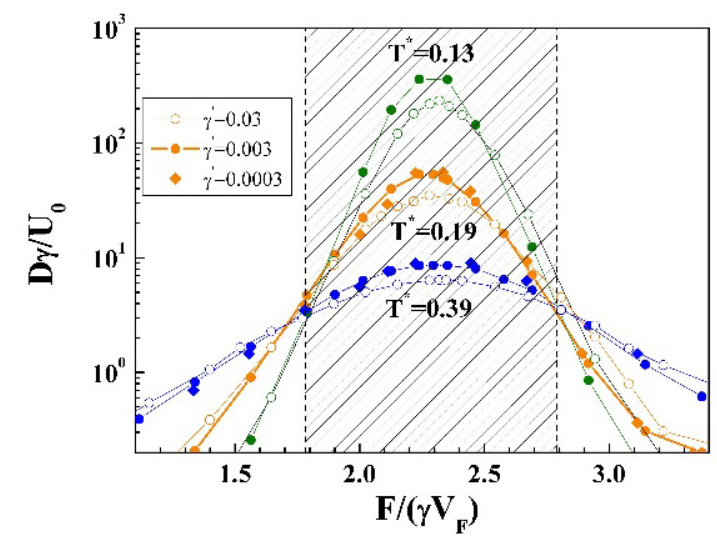

Рис. 3. Зависимость $\mathrm{D} \gamma / \mathrm{U}_{0}$ от $F /\left(\gamma V_{F}\right)$ для различных $\gamma^{\prime}$ и $\mathrm{T}^{*}$. Заштрихована область ТАД.

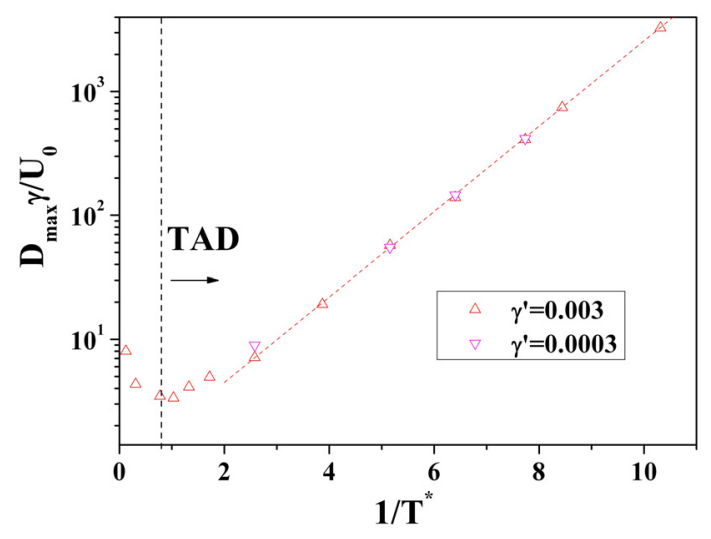

Рис. 4. Зависимость $\mathrm{D}_{\max } \gamma / \mathrm{U}_{0}$ от обратной температуры для различных $\gamma . \nabla-\gamma^{\prime}=3 \cdot 10^{-3}, \Delta-\gamma^{\prime}=3 \cdot 10^{-4}$.

На рис. 4 приведены температурные зависимости коэффициента диффузии при значении силы $F_{m x}$, когда достигается максимальное значение $D_{\max }(T)$ для двух малых значений $\gamma^{\prime}$, отличающихся на порядок. Из графиков видно, что скейлинговая зависимость (9) действительно хорошо выполняется для низких значений $\gamma$.

Из рис. 4 так же можно определить температурный интервал ТАД для недодемпфированных систем. Очевидно, что при температурах, существенно превышающих величину активационного барьера, влияние структуры становиться несущественным. Поэтому движение броуновских частиц можно рассматривать как движение в бесструктурной вязкой среде с трением. В этом случае $D_{\max } \propto T$. Однако при низких температурах, как это видно из рис. $4, D_{\max } \propto \exp (\varepsilon /(k T))$. В работе [33] были найдены аналитические выражения для коэффициентов диффузии частиц в области ТАД. Было показано, что в пределе низких температур действительно $D_{\max } \propto \exp (\varepsilon /(k T))$.

Изменение функциональной зависимости $D_{\max }(T)$ с аномальной $\left(D_{\max } \propto \exp (\varepsilon /(k T))\right.$ ) на нормальную $\left(D_{\max } \propto T\right)$ происходит при значениях температуры $k T \approx U_{0} \quad\left(T^{*} \approx 1\right)$. Как следует из рисунка $\partial D\left(T, F_{T A D}, \gamma\right) / \partial T<0$ только при $k T / U_{0}<1,2$.

Таким образом, в системах с малым трением ТАД наблюдается при температурах $0<k T / U_{0}<1,2$ в интервале сил $1,8<F /\left(\gamma V_{F}\right)<2,8$.

\section{ПЕРЕДЕМПФИРОВАННЫЕ СИСТЕМЫ $(\gamma>2)$}

Для передемфированых систем инерционные эффекты не важны. При этом уравнение (1) упрощается и принимает вид:

$$
\gamma \dot{x}=-\frac{\partial}{\partial x} U(x)+F+\xi(t) .
$$

Численно случай передемпфированых систем был изучен в работах Марчезони [25-26]. Аналитическое решение для температурной зависимости диффузии частиц в этих систем было найдено в работе [35]. На рис. 5 приведена типичная зависимость коэффициента диффузии для передемпфированных систем. Как видно из рисунка, максимальное значение коэффициента диффузии $D_{\max }$ наблюдается при $F=F_{0}$. Рэйманом [35] было показано, что для синусоидального потенциала $D_{\max } \propto T^{1 / 3}$.

На рис. 6 треугольниками $(\Delta)$ приведена полученная численным решением уравнений $(10)$ температурная зависимость $D_{\max }$ для предемпфированых систем. Как видно из рисунка, $D_{\max }$ имеет степенную зависимость $D \propto T^{\alpha}$. При низких температурах $\alpha \approx 0,33$, что согласуется с результатом Рэймана $(\alpha=1 / 3)$. В области высоких температур $\alpha=1$, так как структура среды перестает оказывать влияние на движение броуновских частиц. Изменение показателя степени происходит вблизи температуры $k T \approx 0,5 U_{0}\left(T^{*} \approx 1 / 2\right)$. Хотя диффузия 
усиливается в области низких температур по сравнению с бесструктурной средой, абсолютные значения коэффициента диффузии при низких температурах падают с температурой как $D_{\max } \propto T^{1 / 3}$.

Обратными треугольниками $(\nabla)$ на рис. 6 приведены значения D рассчитанные по уравнениям (1-2) для того же значения $\gamma^{\prime}=2,0$, что и для передемпфированной системы. Как видно из графиков, численные значения, рассчитанные двумя методами, совпадают. Полыми кружками приведены значения $D_{\max }$, для $\gamma^{\prime}=8,0$, которые совпадают с данными для случая $\gamma^{\prime}=2,0$. То есть при $\gamma^{\prime}>2,0$ инерционными эффектами в синусоидальных наклонных потенциалах можно пренебречь, и считать систему передемпфированной.

Таким образом, в передемпфированых системах в синусоидальных наклонных потенциалах зона ТАД отсутствует, так как при любых температурах $\frac{\partial D(T, F, \gamma)}{\partial T}>0$.

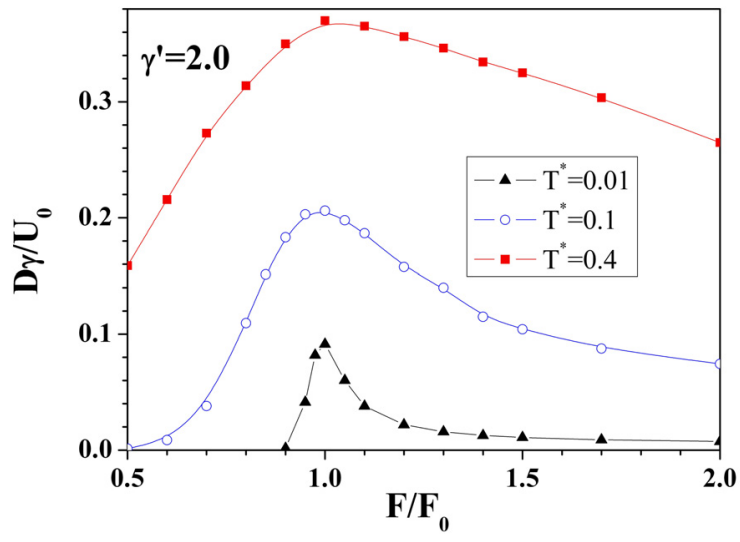

Рис.5. Зависимости коэффициентов диффузии от действующей силы для различных температур. Квадраты $T^{*}=0,4$, кружки $-T^{*}=0,1$, треугольники $-T^{*}=0,01$;

$$
\gamma^{\prime}=2,0 \text {. }
$$

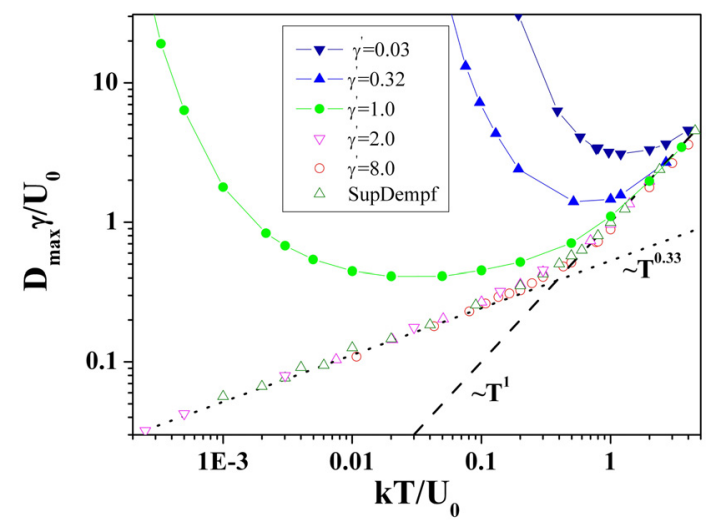

Рис. 6. Зависимость D $\gamma / \mathrm{U}_{0}$ от обратной температуры для различных $\gamma$.

$\Delta-\gamma^{\prime}=2,0$ передемфированные системы, $\nabla-\gamma^{\prime}=2,0$ решение уравнений (1) . Пунктирной линией й нанесена зависимость $T^{1}$, мелким пунктиром зависимость $T^{0.33}$.

ПЕРЕХОДНЫЕ ЗНАЧЕНИЯ КОЭФИЦИЕНТА ТРЕНИЯ $\left(0,03<\gamma^{\prime}<2\right)$

При $\gamma^{\prime}<0,03$ температурный интервал в котором $\partial D\left(T, F_{T A D}, \gamma\right) / \partial T<0$ ограничен температурами, при которых $k T / U_{0}<1,2$. Рассмотрим, как трансформируется температурный интервал ТАД $\Delta T(F)$ с увеличением $\gamma^{\prime}$. На рис. 7 приведена характерная картина зависимости коэффициента диффузии от действующей силы для $\gamma^{\prime}=0,32$. Качественно картина схожа с той, что наблюдается и для случая малой диссипации. В некотором интервале сил коэффициент диффузии возрастает с понижением температуры. Максимальный коэффициент диффузии $D_{\max }\left(T, \gamma^{\prime}\right)$ реализуется при силе $F_{m x}$. Исследуем, как изменяется температурный интервал $\Delta T\left(F_{m x}\right)$ с увеличением трения.

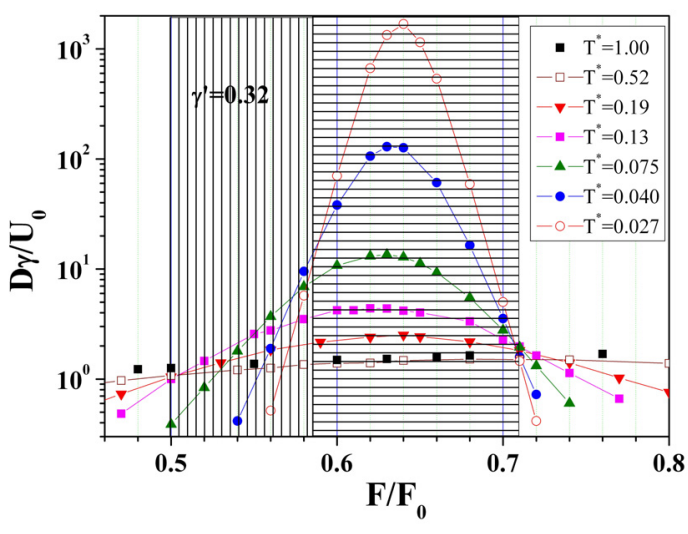

Рис. 7. Зависимость $\mathrm{D} \gamma / \mathrm{U}_{0}$ от $\mathrm{F}$ для различных $\mathrm{T}^{*}$. Горизонтальной штриховкой обозначена низкотемпературная область ТАД. Вертикальная штриховка темпепраурно ограниченной ТАД.

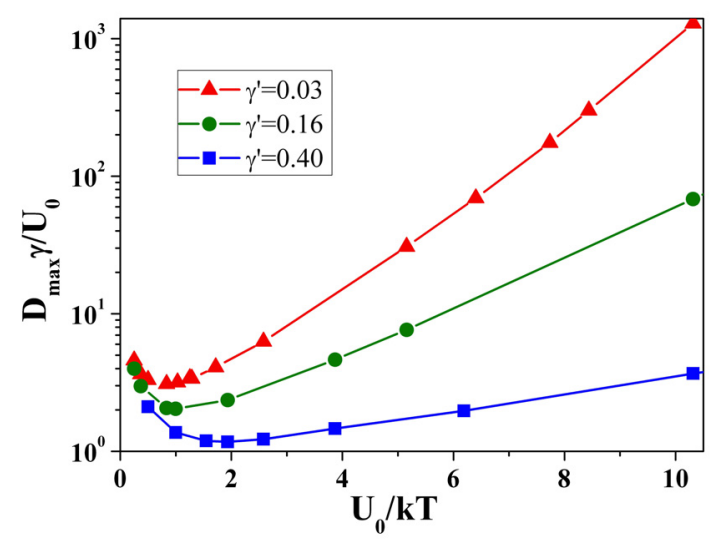

Рис. 8. Зависимость $\mathrm{D} \gamma / \mathrm{U}_{0}$ от обратной температуры для

$$
\gamma^{\prime}=0,03 ; 0,16 ; 0,4 \text {. }
$$


На рис. 8-9 приведены температурные зависимости $D_{\max } \gamma^{\prime} / U_{0}$ от обратной температуры для значений $\gamma^{\prime}$, возрастающих от значения $\gamma^{\prime}=0,03$ до $\gamma^{\prime}=0,95$. Как следует из рис. 8-9, увеличение коэффициента трения приводит к уменьшению температурного интервала ТАД. На рис. 10 приведен график максимальной температуры $T_{T A D}^{*}(\gamma)$, при которой $\frac{\partial D\left(T, F_{m x}, \gamma^{\prime}\right)}{\partial T}<0$.

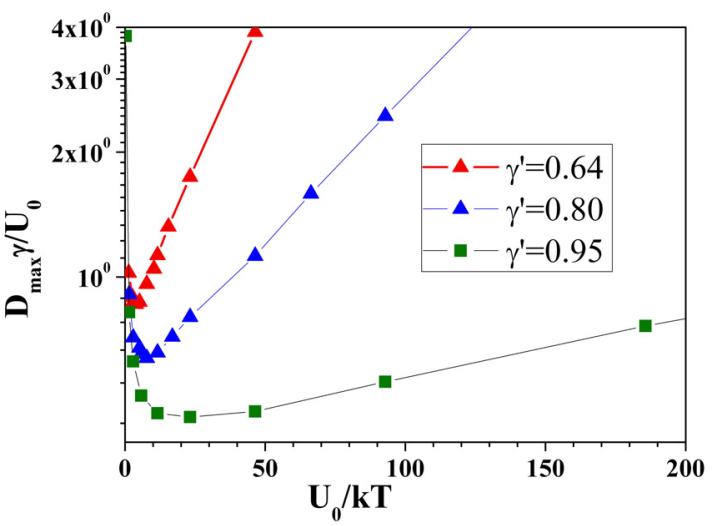

Рис. 9. Зависимость $\mathrm{D} \gamma / \mathrm{U}_{0}$ от обратной температуры для $\gamma^{\prime}=0,64 ; 0,8 ; 0,95$.

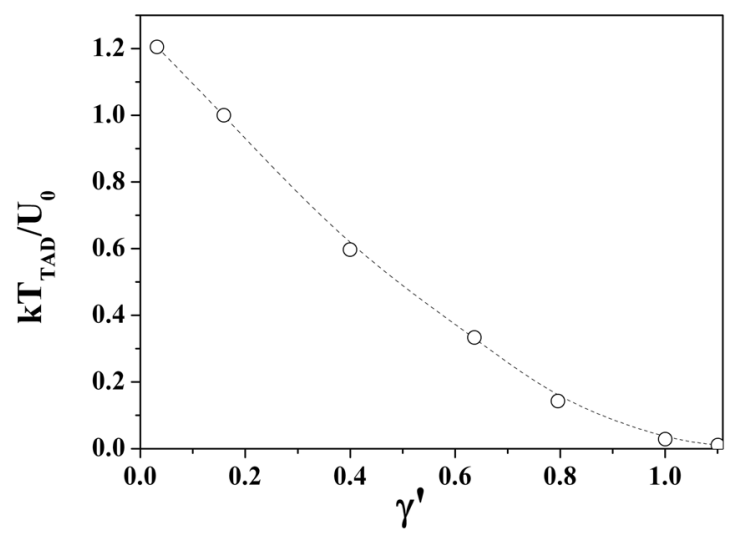

Рис. 10. Зависимости верхней границы температурного интервала ТАД от величины $\gamma^{\prime}$ при действующей силе

$$
F_{m x}\left(\gamma^{\prime}\right) \text {. }
$$

Видно, что при $\gamma^{\prime}>1$ температура верхней границы ТАД близка к нулю. При $\gamma^{\prime}=1,1$ температура $T_{T A D}^{*}=0,01$. Таким образом, с увеличением $\gamma^{\prime}$ температурно-аномальная диффузия исчезает, так как сужается температурный интервал, в котором наблюдается ТАД.

Как видно из рис. 8-9, для рассматриваемых значений $\gamma^{\prime}$, так же как и для малых коэффициентов трения, $D_{\max } \propto \exp \left(\varepsilon_{m x} /(k T)\right)$, где $\varepsilon_{m x}$ - положительная величина. Однако само значение $\varepsilon_{m x}$ уменьшается с $\gamma^{\prime}$. Это изменение связано с изменением потенциального рельефа, в котором движется броуновская частица. В отсутствие внешней силы $(\mathrm{F}=0)$ минимальное значение потенциала наблюдается в точках $x=2 \pi N$, где $N$ целое число. А максимум - при $x=\pi+2 \pi N$. При этом активационный барьер $\Delta U$, который преодолевают частицы при переходе из одной ячейки в другую, равен $U_{0}$. С увеличением $\gamma^{\prime}$ возрастает $F_{m x}$. Активационный барьер при этом уменьшается, как это схематично показано на рис. 11 для действующей силы $F / F_{0}=0,4$. Минимальное значение $U(x)$ теперь реализуется при $x=\operatorname{Arcsin}(F)+2 \pi N$, а максимальное - при $x=\pi-\operatorname{Arcsin}(F)+2 \pi N$. И соответственно $\Delta U(0)>\Delta U(0,4)$.

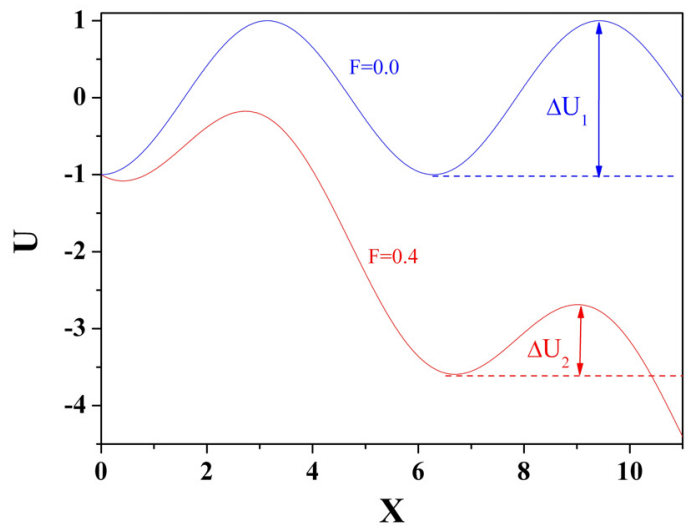

Рис. 11. Зависимости величины активационного барьера $\Delta U$ от величины силы $F$.

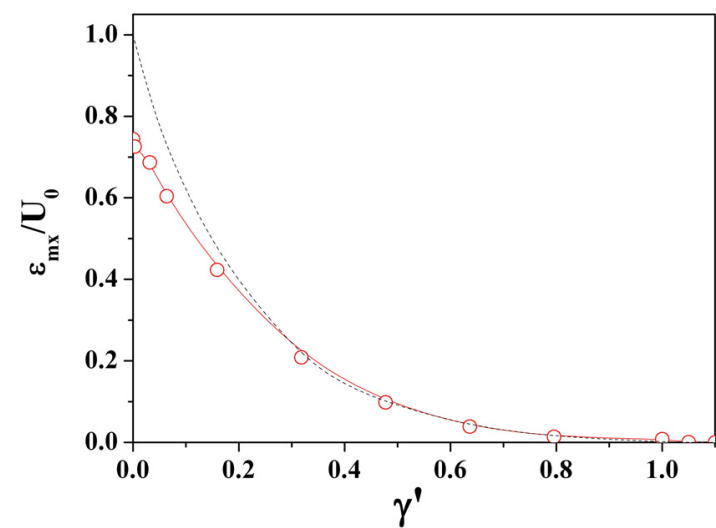

Рис. 12. Зависимости величины барьера $\varepsilon_{m x}$ от величины $\gamma^{\prime}$. Пунктирной линией показано изменение потенциального барьера $\Delta U(\gamma)$ в периодическом наклонном потенциале.

Уменьшение барьера в Х-пространстве приводит к уменьшению барьера в пространстве скоростей и соответственно к уменьшению $\varepsilon_{m x}(\gamma)$. Величины $\varepsilon_{m x} / U_{0}$ представлены на рис. 12 полыми маркерами. 
Пунктирной линией показано изменение потенциального барьера $\Delta U\left(F_{m x}\left(\gamma^{\prime}\right)\right)$ в пространственной решетке. Видно, что зависимости $\Delta U(\gamma)$ и $\varepsilon_{m x}(\gamma)$ хорошо коррелируют между собой при $\gamma^{\prime}>0,3$. Аналогично с $T_{T A D}$, при $\gamma^{\prime}>1,1$ исчезает экспоненциальная зависимость ТАД от обратной температуры.

Теперь рассмотрим, каким образом изменяется ширина силового интервала зоны ТАД $\Delta F$ с изменением $\gamma^{\prime}$. Из рис. 7 видно, что для промежуточных значений $\gamma$, так же как и для случая малых $\gamma$ в ограниченном интервале сил существует зона ТАД. Однако в случае переходных значений $\gamma$ наблюдаются особенности. Слева на рис. 7 вертикальной штриховкой приведена область ограниченной ТАД. В этой области при фиксированном значении силы диффузия усиливается с ростом температуры вплоть до некоторого значения температуры, а затем начинает уменьшаться. Горизонтальной штриховкой на рис. 7 показана область, в которой в исследованном диапазоне температур с уменьшением Т коэффициент диффузии всегда возрастает.

На рис. 13 приведены зависимости $\mathrm{D}$ от обратной температуры для трех различных значений действующей силы из рис. 7.

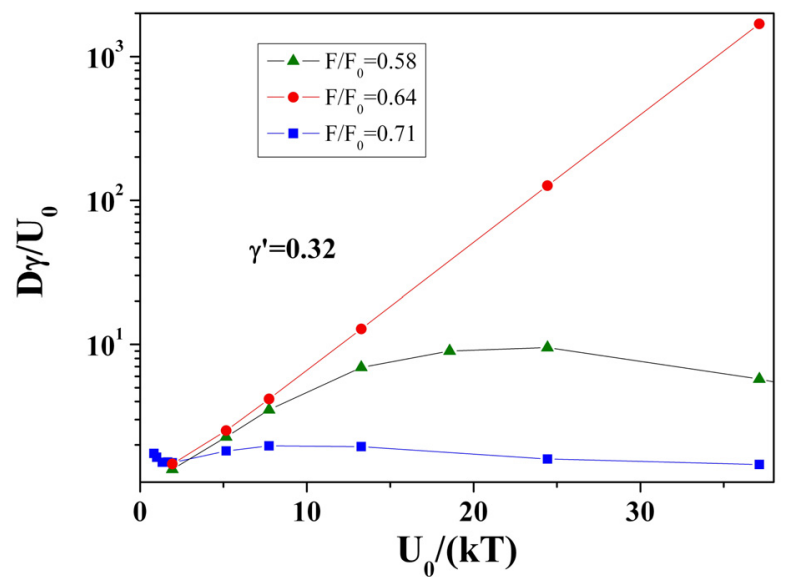

Рис. 13. Зависимость $\mathrm{D} \gamma / \mathrm{U}_{0}$ от обратной температуры для 3 фиксированных значений силы: $\mathrm{F}^{\prime}=0,58$ (треугольники), $\mathrm{F}^{\prime}=0,71$ (квадраты) и $\mathrm{F}^{\prime}=0,64$ (кружки). $\gamma^{\prime}=0,32$.

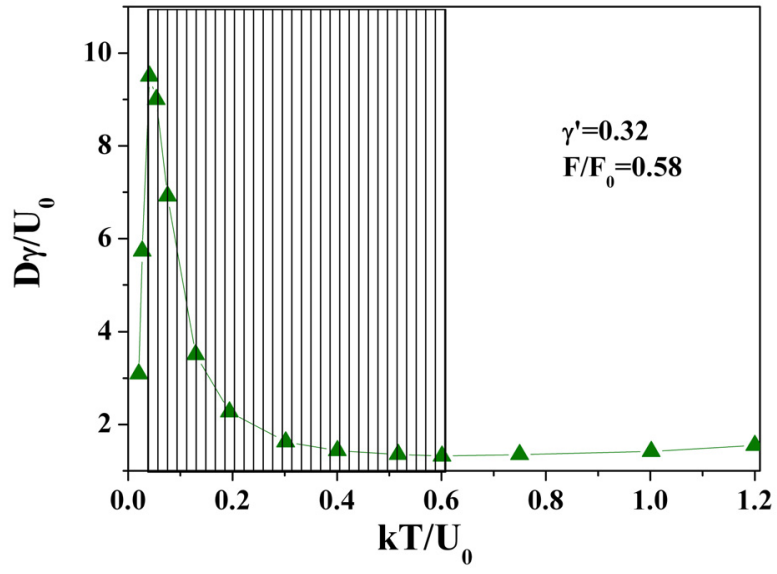

Рис. 14. Зависимость $\mathrm{D} \gamma / \mathrm{U}_{0}$ от температуры для значения силы $\mathrm{F}^{\prime}=0,58, \gamma^{\prime}=0,32$.

Штриховкой выделено температурное “окно” ТАД.

Значение $F / F_{0}=0,64$ соответствует максимальному значению коэффициента диффузии. Как видно из рис. 13, в области низких температур величина $D_{\max } \propto \exp \left(\varepsilon_{m x} /(k T)\right)$ так же как и в случае малых $\gamma^{\prime}$. Однако при $F^{\prime}=0,58$ коэффициент диффузии в низкотемпературной области сначала возрастает с уменьшением температуры, а затем начинает падать. То есть возникает температурное “окно” ТАД. На рис. 14 эта область выделена вертикальной штриховкой. Из рис. 14 следует, что $\partial D / \partial T<0$ лишь в интервале температур $0,04<k T / U_{0}<0,6$. Возможность такого рода зависимости обсуждалась в работе Б. Лиднера и И. Соколова [37]. На основании аппроксимации зависимости скорости переходов между “бегущими” и “локализованными” состояниями, они пришли к выводу о существовании ограниченного снизу по температуре интервала ТАД. Рис. 14 показывает, что такая зависимость действительно реализуется. В настоящее время мы не можем сказать, будет ли зона ТАД сужаться до полного исчезновения с уменьшением температуры, или существует низкотемпературный предел ширины интервала сил, как и для систем с малыми $\gamma^{\prime}$.

На рис. 7 так же обращает на себя внимание тот факт, что в отличие от случая малых значений $\gamma^{\prime}$ поведение температурных кривых диффузии слева и справа от значения $F_{m x}$ различно. На левой границе ТАД существует широкий интервал сил, в котором наблюдается температурное “окно” ТАД. Однако на правой границе эта область практически отсутствует. Так при $F^{\prime}=0,71$ коэффициент диффузии слабо зависит от температуры, как это видно из рис. 13.

Такие же зависимости, как и на рис. 7 были построены для целого ряда переходных значений коэффициентов диффузии. Так на рис. 15 в качестве примера приведены данные для $\gamma^{\prime}=1,0$. Видно, что величина $\Delta F(\gamma)$ уменьшилась по сравнению с $\gamma^{\prime}=0,32$. При этом по-прежнему на левой границе ТАД существует участок температурного “окна”. Однако, в отличие от случая с $\gamma^{\prime}=0,32$, такой же участок симметрично относительно центра существует и на правой границе. На рис. 16 представлены температурные зависимости для различных значений действующей силы. Из графиков хорошо видно наличие температурного “окна" на границе $\Delta F(1,0)$. 


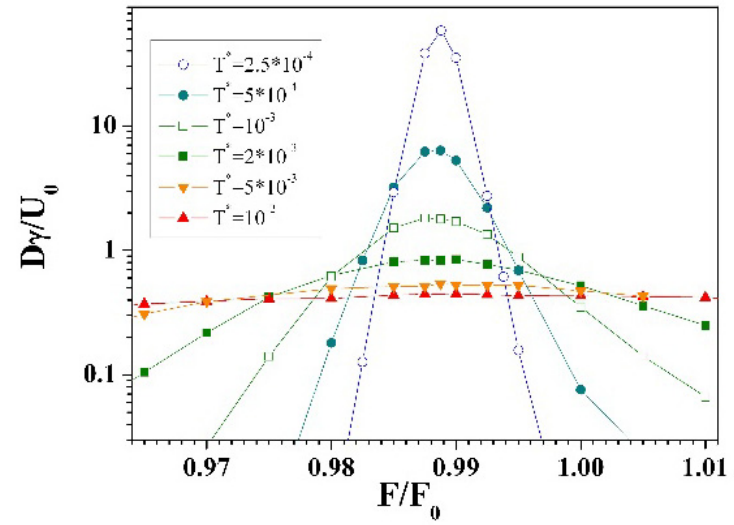

Рис. 15. Зависимость D $\gamma / \mathrm{U}_{0}$ от силы для различных значений температуры.

$\gamma^{\prime}=1,0$

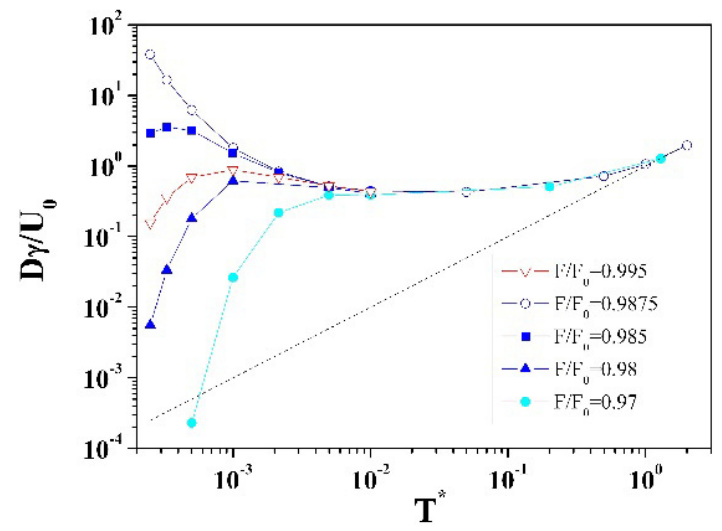

Рис. 16. Зависимость $\mathrm{D} \gamma / \mathrm{U}_{0}$ от температуры для различных значений силы. $\gamma^{\prime}=1,0$

Пунктирной линией показан коэффициенты диффузии в вязкой среде: $D=k T / \gamma$.

На рис. 17-18 приведена итоговая картина изменения интервала существования ТАД $\Delta F\left(\gamma^{\prime}\right)$. На рис. 17 маркерами приведены значения ширины силового интервала $\Delta F\left(\gamma^{\prime}\right)$, в котором наблюдалось $\partial D / \partial T<0$ хотя бы для какой-нибудь температуры. На рис. 18 заполненными маркерами приведены минимальные и максимальные значения сил, при котором наблюдалось ТАД. Полыми маркерами приведены значения силы, при которой коэффициент диффузии максимален для данного коэффициента трения. Видно, что для $0,2<\gamma^{\prime}<0,5$ значение $F_{m x}$ ассиметрично относительно концов отрезка существования ТАД. При $\gamma^{\prime}>1,1$ ширина силового интервала, в котором наблюдется $\partial D / \partial T<0$, стремится к нулю.

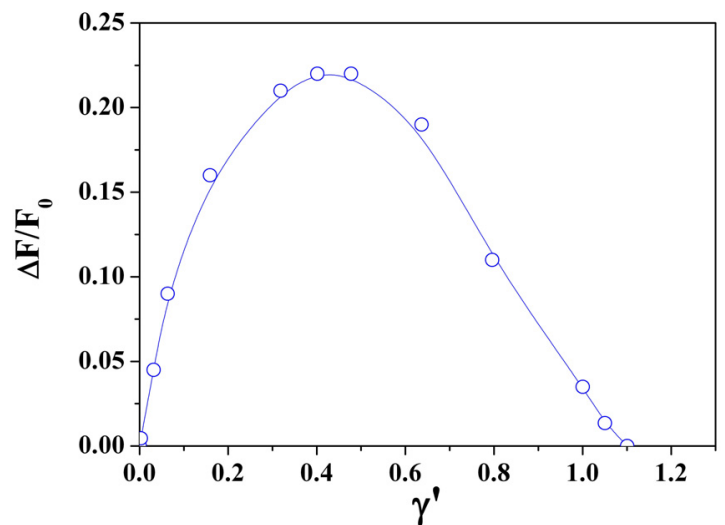

Рис. 17. Изменение ширины зоны ТАД в зависимости от коэффициента трения.

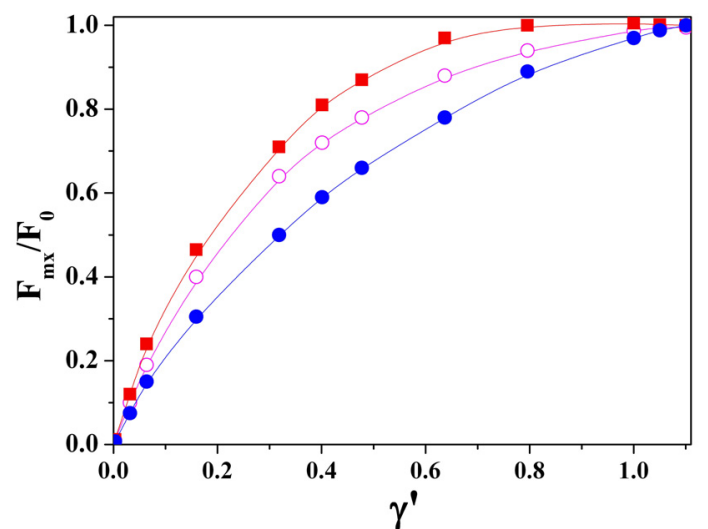

Рис. 18. Диаграмма зоны ТАД. Кружками обозначено нижнее значение области возникновения ТАД (при любых Т), квадратами - значения при которых ТАД исчезает.

Незаполненными кружками отмечены значения при которых реализуется максимальная диффузия.

\section{ВЫВОДЫ}

В работе изучена диффузия частиц в наклонных пространственно-периодических потенциалах в широком диапазоне температур. Исследованы системы с различным уровнем коэффициента трения $\gamma^{\prime}$. Показано, что как в недодемпфированых, так и в передемпфированых системах, коэффициент диффузии достигает максимального значения $D_{\max }$ при определенном значении внешней силы, величина которой зависит от $\gamma^{\prime}$. Однако в системах с малым и большим трением температурная зависимость $D_{\max }$ различается. В недодемпфированных системах в низкотемпературной области наблюдается температурно-аномальная диффузия (ТАД), при которой $D$ возрастает с понижением температуры экспоненциально с обратной температурой: $D \propto \exp (\varepsilon / k T)$. ТАД 
наблюдается при температурах $0<k T / U_{0}<1,2$ в интервале сил $1,8<F /\left(\gamma V_{F}\right)<2,8$. В системах с большим трением ТАД отсутствует. Однако изменяется показатель $\alpha$ в степенной зависимости коэффициента диффузии с ростом температуры: $D \propto T^{\alpha}$. При $T^{*}<0,5$ показатель $\alpha=1 / 3$ для синусоидального потенциала, а при $T^{*}>1$ показатель $\alpha=1$.

В работе исследовано, каким образом осуществляется переход от экспоненциальной зависимости ТАД к обычной степенной температурной зависимости с ростом $\gamma^{\prime}$. Показано, что с увеличением коэффициента трения энергетический барьер $\varepsilon$ разделяющий “бегущие” и “локализованные” решения уменьшается. При $\gamma^{\prime}>1.1$ величина $\varepsilon$ стремится к нулю.

Исследования показали, что в области промежуточных значений коэффициента трения $0,1<\gamma^{\prime}<2$ возникает температурное “окно” ТАД. В некотором интервале сил коэффициент диффузии сначала возрастает с уменьшением температуры, а затем снова начинает падать. Построены диаграммы существования таких областей.

Полученные результаты открывает перспективы создания новых технологий управления процессами диффузии. Это имеет большое значение для получения наноматериалов с заданной структурой, создания поверхностных наноструктур и пр.

\section{ORCID IDs}

I.G. Marchenko (D) https://orcid.org/0000-0003-1341-4950, I.I. Marchenko (D) https://orcid.org/0000-0002-3071-9169,

V.I. Tkachenko (D) https://orcid.org/0000-0002-1108-5842

\section{СПИСОК ЛИТЕРАТУРЫ}

[1]. A.N. Gan'shin, V.N. Grigor'ev, V.A. Maidanov, N.F. Omelaenko, A.A. Penzev, E.Ya. Rudavskii and A.S. Rybalko, Low Temperature Physics. 25, 259 (1999), doi: 10.1063/1.593738.

[2]. R. Salgado-Garcia, Phys. Rev. E. 90, 032105 (2014).

[3]. J. Spiechowicz, P. Talkner, P. Hänggi and J. Luczka, New J. Phys. 18, 123029 (2016).

[4]. J. Spiechowicz, M. Kostur and J. Luczka, Chaos. 27, 023111 (2017).

[5]. D. Speer, R. Eichhorn and P. Reimann, Europhys. Lett. 97, 60004 (2012).

[6]. E. Heinsalu, T. Örd and R. Tammelo. Phys. Rev. E. 70, 041104 (2004).

[7]. J. Spiechowicz, J. Luczka, Phys. Rev. E. 91, 062104 (2015).

[8]. E.Heinsalu, R. Tammelo and T. Ord, Phys. Rev. E. 69, 021111 (2004).

[9]. D. Dan, A. M. Jayannavar, Phys. Rev. E. 66, 041106 (2002).

[10]. L. Angelani, G. Foffi, F. Sciortino and P. Tartaglia, J. Phys. Condens. Matter. 17, L113-L119 (2005).

[11]. B. Lindner, M. Kostur and L. Schimansky-Geier, Fluct. and Noise Let. 1, R25-R39 (2001).

[12]. C.K. Lee, J. Moix and J. Cao, Journ. Chem. Phys. 142, 164103 (2015).

[13]. M. Guo, H. Gelman and M. Gruebele, PLoS ONE. 9, e113040 (2014).

[14]. W.S. Tung, P.J. Griffin, J.S. Meth, et al., ACS Macro Lett. 5, 735-739 (2016).

[15]. V.B. Eltsov, A.P. Finne, R. Ha“nninen, et al., Phys. Rev. Lett. 96, 215302 (2006).

[16]. Ch.K. Lee, J. Moix and J. Cao, Chem. Phys. 142, 164103 (2015).

[17]. S. Iubini, O. Boada, Y. Omar and F. Piazza, New J. Phys. 17, 113030 (2015).

[18]. H. Risken, The Fokker-Planck Equation and Methods of Solution and Applications (Springer, 1989), p. 472.

[19]. H. Risken, H.D. Vollmer, Phys. Lett. 69. A, 387-389 (1979).

[20]. H. Risken, H.D. Vollmer, Z. Physik B. 33, 297 (1979).

[21]. H. Risken, H.D. Vollmer, Z. Physik B. 37, 343-349 (1980).

[22]. H. Risken, H.D.Vollmer, Z. Physik B. 34, 313-322 (1979).

[23]. H. Risken, H.D.Vollmer, Z. Physik B. 35, 177-184 (1979).

[24]. H. Risken, H.D.Vollmer, Z. Physik B. 54 357-370 (1984).

[25]. G. Costantini, F. Marchesoni, Europhys. Lett. 48, 491-497 (1999).

[26]. M. Borromeo, G. Costantini and F. Marchesoni, Phys. Rev. Lett. 82, 2820-2823 (1999).

[27]. K. Lindenberg, J.M. Sancho, A.M. Lacasta and I.M. Sokolov, Phys. Rev. Lett. 98, 020602 (2007).

[28]. J. M.Sancho, A.M. Lacasta, K. Lindenberg, I.M. Sokolov and A.H. Romero, Phys. Rev. Lett. 92, 250601 (2004).

[29]. M. Khoury, J. P. Gleeson, J. M. Sancho, A. M. Lacasta and K. Lindenberg, Phys. Rev. E. 80, 021123 (2009).

[30]. K. Lindenberg, A.M. Lacasta, J.M. Sancho and A.H. Romero, New Jour. of Phys. 7, 29 (2005).

[31]. I.G. Marchenko, I.I. Marchenko, Europhys. Let. 100, 5005 (2012).

[32]. I.G. Marchenko, I.I. Marchenko and A.V. Zhiglo, Europ. Phys. Jour. B. 8710 (2014).

[33]. I.G. Marchenko, I.I. Marchenko and V.I. Tkachenko, JETP Let. 106, 242-246 (2017).

[34]. I.G. Marchenko, I.I. Marchenko and A.V. Zhiglo, Phys. Rev. E. 97, 012121 (2018).

[35]. P. Reimann, C. Van den Broeck, H. Linke, P. Hänggi, J.M. Rubi, and A. Pérez-Madrid, Phys. Rev. E. 65, 031104 (2002).

[36]. K. Lindenberg, J.M. Sancho, A.M. Lacasta and I.M. Sokolov, Phys. Rev. Lett. 98, 020602 (2007).

[37]. B. Lindner, I.M. Sokolov, Phys. Rev. E. 93, 042106 (2016).

[38]. I.G. Marchenko, I.I. Marchenko, JETP Let. 95, 137-142 (2012).

[39]. D.G. Alciatore and M.B. Histand, Introduction to Mechatronics and Measurement Systems (McGraw Hill, 2007 ), p. 553. 